\title{
CLINICO-PATHOLOGICAL FEATURES AND TREATMENT PARTICULARITIES OF INCIDENTALLY DIAGNOSED ENDOMETRIAL ADENOCARCINOMA IN INFERTILE WOMEN
}

\author{
O. Kaabia, L. Aymen, G. Nourallah, Z. Rym, S. Aymen, B. Mohamed, K. Hédi . \\ Université de Sousse- Faculté de Médecine de Sousse- Hopital Farhat Hached- \\ LR12ES03- 4000- Sousse - Tunisia,
}

\section{Introduction:}

Endometrial cancer (EC) is a common gynecologic malignant condition in developing countries in menopausal women. It is rare in young women with a prevalence of almost $5 \%$ in women under the age 45 . The aim of this study was to investigate the clinical and prognostic features of women under 45 with the incidental diagnosis of endometrial adenocarcinoma during the infertility work-up, with special attention given to the treatment approaches and the survival rates.

\section{Methods:}

The medical records of 228 patients who were diagnosed with EC at the department of Gynecology and Obstetrics in Farhat Hached Teaching Hsopital Sousse Tunisia, between 2008 and 2018, were included in the study and analyzed retrospectively. Out of these patients, $9.6 \%(n=22)$ were $\leq 45$ years.

Eleven patients had a history of infertility and had been diagnosed with EC during a hysteroscopic evaluation for infertility. We compared these patients to the other eleven patients diagnosed with EC under 45 with no infertility history.

\section{Results:}

The age and medical history of the patients in each group are represented in the table 1.

The mean duration of infertility was $5.4 \pm 2.1$ years.

There were no significant difference in terms of early stage cancer ( $p=0.79$ ) between the two groups (Respectively, $81.81 \%$ versus $90.90 \%$ in the fertility and infertility groups) .

A fertility sparing treatment was initiated in 9 patients but after 6 months only 3 had a complete pathological response.

There was no statistical difference in DFS and OS at 5 years between the 2 groups (respectively, $p=0.48$ et $p=0.97$ ).

\section{Discussion :}

During the infertility work-up espetially in women over 40 years old, the endovaginal pelvic ultrasound is necessary in order to asses the thickness of the of the endometrium. The main goeal is to assess the normality of the endometrium but in case of abnormality and beside the diagnosis of endometrial polyps and myomas, doctors have to keep in mind precancerous and cancerous endometrial lesions. This emphasis the interest of systematic hysteroscopy with endometrial biopsies in case of any endometrial abnormality in infertile women over 40 . I

$\mathrm{n}$ fact, the diagnosis of asymptomatic endometrial cancer in young infertile women must not be ruled out without thorogh evaluation.

Howerver in case of an endometrial cancer in young infertile women, a conservative treatement can be prescribed in order to preserve the fertility and a fertility sparing protocol must be discussed with the patient in order to inform her of her options. If accepted and feasable, the endomettrectomy associated with intra-uterine levonorgestrel divice is a seductive option but it has not yet proven its efficiency with very modest results.

In case of association of a pregnancy to a completely resected stage la well differenciated endometriod adenocarcinoma there is no clear proven strategy.

\section{Conclusion :}

The investigation of the endometrium during infertility work-up may lead to the diagnosis of asymptomatic EC that does not seem to have any specific prognostic features even though it may allow a fertility sparing treatment.

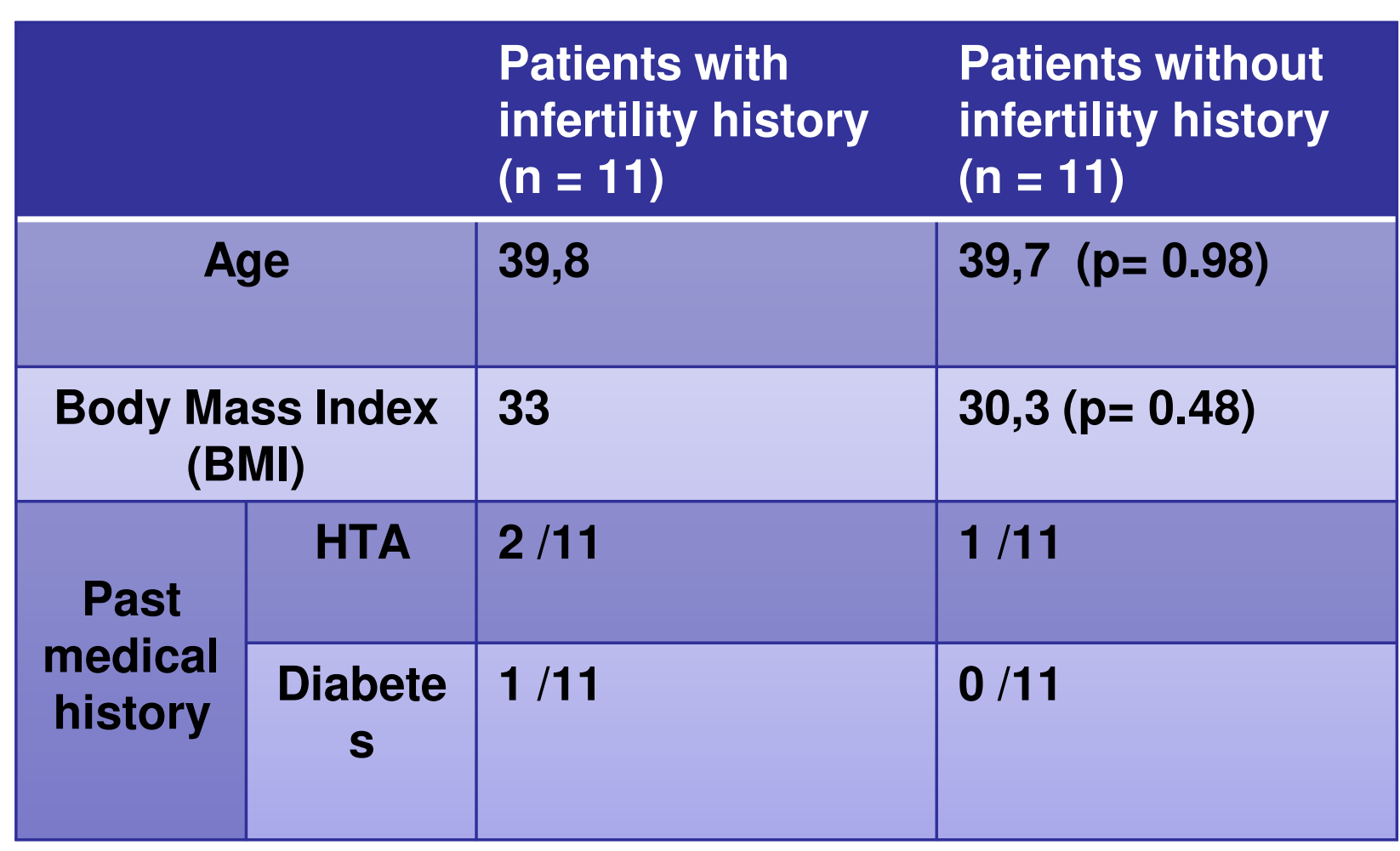

Table 1:

The risk factors of the endometrial cancer 